\title{
Factores de riesgo asociados con la presencia de cerdos fatigados durante el transporte y sus implicaciones en el pH muscular alto
}

\author{
Risk factors associated with the presence of fatigued pigs during transport and \\ their implications on high $\mathrm{pH}$ in muscle
}

Marlyn Hellen Romero ${ }^{1,2}$, Alexander Castillo-Patiño ${ }^{1}$, Jorge Alberto Sánchez ${ }^{1}$

\section{Resumen}

\begin{abstract}
El objetivo del presente estudio fue determinar los factores de riesgo asociados con la presencia de cerdos fatigados durante el transporte y sus implicaciones en el $\mathrm{pH}$ muscular alto en cerdos de ceba comercial. Se realizó un estudio de casos y controles, que incluyó 94 cerdos fatigados como casos y 94 cerdos típicos como controles. Los cerdos utilizados en este estudio eran machos castrados y hembras jóvenes de un genotipo comercial y peso de mercado estándar (115.5 $\pm 1.3 \mathrm{~kg}$ en promedio). Se aplicaron encuestas estructuradas para caracterizar las condiciones de transporte y se evaluó el $\mathrm{pH}_{45}$ y $\mathrm{pH}_{24}$ de las canales. El modelo de regresión logística mostró que el número de compartimientos, la presencia de asistente, el sexo, la velocidad y el tiempo de transporte fueron variables asociadas con la presencia de cerdos fatigados. Los cerdos fatigados presentaron mayor probabilidad de $\mathrm{pH}$ muscular alto que los cerdos normales $(\mathrm{p}<0.01)$. Estos resultados indican que el transporte es un factor de estrés para los cerdos con implicaciones sobre la presencia de cerdos fatigados y carne con $\mathrm{pH}_{24}$ alto.
\end{abstract}

Palabras clave: bienestar animal; calidad de la carne; pérdidas por transporte

\section{AbstraCT}

The aim of this study was to determine the transport risk factors associated with the presence of fatigued pigs and its implications on high muscle $\mathrm{pH}$ in market-weight pigs. A case-control study was conducted, including 94 fatigued pigs as case and 94 typical pigs as controls. Pigs used in this study were castrated males and young females of a commercial genotype and standard market weight (mean BW $115.5 \pm 1.3 \mathrm{~kg}$ ) castrated

\footnotetext{
${ }^{1}$ Departamento de Salud Animal, Facultad de Ciencias Agropecuarias, Universidad de Caldas, Manizales, Colombia

${ }^{2}$ E-mail: marlyn.romero@ucaldas.edu.co
}

Recibido: 6 de abril de 2018

Aceptado para publicación: 20 de septiembre de 2018 
and gilts of a standard commercial genotype. Structured face-to-face questionnaires were applied to characterize the transport conditions, and $\mathrm{pH}_{45}$ and $\mathrm{pH}_{24}$ carcass were evaluated. The logistic regression model showed that numbers of decks, the presence of assistant, sex, and transport speed and time were variables associated with the presence of fatigued pigs. Fatigued pigs had more probability of high muscular $\mathrm{pH}$ than typical pigs $(p<0.01)$. These results indicate that the transport is a stress factor for pigs with implications on the presence of fatigued pigs and meat with high $\mathrm{pH}_{24}$.

Key words: transport losses; animal welfare; meat quality

\section{INTRODUCCIÓN}

El transporte es un proceso inevitable en la industria porcina moderna, que representa una fase crítica en la producción animal y se considera a menudo como una de las principales causas de estrés para los animales de granja (Magnani et al., 2014). La mayoría de los cerdos de ceba son transportados en camiones desde la granja hasta la planta de sacrificio, donde gran parte de ellos llega y se procesa sin incidentes. Sin embargo, un pequeño porcentaje de animales mueren y otros presentan dificultad para caminar durante el desembarque, lo que se conoce comúnmente como «cerdos no ambulatorios» (Sutherland et al., 2009a; Johnson et al., 2013). La presencia de cerdos no ambulatorios o que mueren en cualquier etapa durante el proceso de comercialización se denominan «pérdidas por transporte» (Berry et al., 2012).

Los cerdos fatigados exhiben un comportamiento alterado caracterizado por la presencia de dificultad respiratoria (jadeo), decoloración de la piel, o ambos. Si el estrés no se elimina o si se encuentran factores estresantes adicionales, el cerdo se mostrará reacio a moverse, con vocalizaciones anormales, desarrollo de temblores musculares o manifestará una combinación de indicadores de estrés (Ritter et al., 2009a; Johnson et al., 2013). Los cerdos fatigados presentan acidosis metabólica como respuesta al manejo y al transporte (Ritter et al., 2006), con- dición que puede dar como resultado una reducción de 0.5 unidades del $\mathrm{pH}$ sanguíneo y aumento de la temperatura corporal de 1.0$2.5^{\circ} \mathrm{C}$ (Aradom et al., 2012). Dependiendo del tiempo previo al sacrificio en donde se presente el estrés, este estado puede conducir a carne pálida, suave y exudativa (PSE) $\mathrm{u}$ oscura, firme y seca (DFD) (Carr et al., 2005). La variación en el pH influye en las características de la calidad de la carne, como el color, la vida útil, la capacidad de retención de agua y los rendimientos tecnológicos (Gajana et al., 2013).

Los cerdos fatigados representan un problema para la industria porcina por su impacto negativo en el bienestar de los animales, las pérdidas económicas y las implicaciones en la calidad de la carne (Kephart et al., 2010). Las pérdidas por transporte en Estados Unidos representan un 30\% del valor total del animal, por los decomisos relacionados con la presencia de hematomas y animales muertos, tiempo y mano de obra adicional, y una mayor capacitación del personal, entre otros (Carr et al., 2005; Ritter et al., 2009b). Sin embargo esta información no incluye los posibles impactos en la calidad de la carne.

Colombia es el quinto mayor productor latinoamericano de carne de porcino con una demanda comercial de 4.2 millones de cabezas (FAOSTAT, s.f.). No obstante, la incidencia de cerdos fatigados en Colombia ha sido escasamente documentada. Se dispone del reporte de Romero et al. (2016) quienes 
establecieron que el $0.4 \%$ de cerdos transportados correspondió a cerdos fatigados. El objetivo del presente estudio fue determinar los factores de riesgo asociados con la presencia de cerdos fatigados durante el transporte y sus implicaciones en el $\mathrm{pH}$ muscular alto en cerdos de ceba en una planta comercial colombiana.

\section{Materiales y Métodos}

El estudio se llevó a cabo en una planta de sacrificio comercial localizada en la región andina de Colombia entre febrero y abril de 2015. La planta procesa en promedio 350 porcinos por día.

\section{Consideraciones Éticas}

Los protocolos y procedimientos empleados en esta investigación contaron con la aprobación del Comité de Ética para la Experimentación con Animales (CEEA) de la Universidad de Caldas (Acta 1 07/05/2014 Actividades con riesgo mínimo), Manizales, Colombia. La planta de sacrificio comercial cumplió con el decreto 1500 (MPS, 2007). Los animales fueron transportados y sacrificados de acuerdo con las regulaciones nacionales aplicadas en investigación y sacrificio comercial.

\section{Manejo de los Cerdos en la Planta de Sacrificio}

Los cerdos se desembarcaron en la planta de sacrificio por medio de una rampa metálica de pendiente ajustable ( $8 \mathrm{~m}$ de longitud) con piso antideslizante y se alojaron en corrales a una densidad de carga de $1 \mathrm{~m}^{2} /$ animal durante 22 horas, con suministro de agua ad libitum y sin mezcla social. Todos los cerdos fueron sometidos a aturdimiento eléctrico de un punto usando $250 \mathrm{v}$ y 1.3 A durante 3 segundos. Luego se izaron, desangraron y se sometieron a escaldado a $62^{\circ} \mathrm{C}$ durante 5 minutos. Las canales se refrigeraron a $4{ }^{\circ} \mathrm{C}$ por 24 horas.

\section{Población de Estudio y Criterios de In- clusión}

Se realizó un estudio de casos y controles que incluyó 94 cerdos fatigados como casos y 94 cerdos típicos (normales) como controles, de acuerdo con el cálculo del tamaño de la muestra a priori (potencia $=0.8$, proporción casos: controles $=1$, precisión $=$ 0.95 , probabilidad de exposición entre controles $=0.30$, razón de disparidad [OR] detectada $=2$ ). Se seleccionaron cerdos castrados de ceba (peso promedio $115.5 \pm 1.3$ $\mathrm{kg}$ ) y cerdas jóvenes de un genotipo comercial estándar. Los cerdos fatigados se definieron como cerdos sin lesiones, traumas o enfermedades obvias, pero incapaces de caminar o mantenerse con sus congéneres y que mostraban signos físicos de estrés (disnea, decoloración de la piel, vocalización anormal y temblores musculares) (Ritter et al., 2006). Los cerdos típicos (normales) se definieron como cerdos ambulatorios sin lesiones, traumas o enfermedades obvias, que podían caminar o mantenerse con sus congéneres y sin signos físicos de estrés.

Los cerdos fatigados se identificaron usando números de tatuaje únicos y se alojaron en el corral de observación, hasta que el inspector oficial autorizó su sacrificio. Los cerdos de ceba típicos se identificaron y se localizaron en los corrales de recepción.

\section{Recopilación de Datos}

Los datos se obtuvieron a partir de evaluación visual y encuestas estructuradas presenciales dirigidas a los conductores de los camiones, que incluyó información sobre las condiciones de transporte y factores demográficos.

\section{Medición de pH}

Se utilizó un medidor de $\mathrm{pH}$ portátil, equipado con un electrodo penetrable (IQ150 $\mathrm{pH} / \mathrm{Mv} /$ medidor de temperatura; IQ Scientific Instruments) para determinar el $\mathrm{pH}_{45} \mathrm{y} \mathrm{pH}_{24}$. El electrodo se insertó en una 
pequeña incisión en el músculo longissimus en el lado izquierdo de la canal $\left(3^{\circ} / 4^{\circ}\right.$ último nivel de costilla). Después de cada cinco muestras, el medidor de $\mathrm{pH}$ se recalibró a temperatura ambiente utilizando dos soluciones tampón estándar de pH 7.0 y 4.0.

\section{Manejo de Datos y Análisis Estadísticos}

Los análisis de los datos se realizaron por medio de un modelo de regresión logística univariable, con el software STATA v. 13.0 para Windows (College Station, EEUU). Se utilizó el tamaño del grupo en el camión como un factor de confusión a priori, para determinar si cada variable se asociaba significativamente con el estado de casos y controles (variable dependiente) para la progresión al análisis multivariable. Se realizó una regresión logística multivariable, considerándose para su inclusión variables con valores de $\mathrm{p}<0.20$ en el análisis univariable (basado en la prueba de la relación de semejanza). Las variables no significativas se eliminaron una por una del modelo, comenzando con la variable que mostró el mayor valor de $\mathrm{p}$ general.

El modelo se volvió a ejecutar y se evaluó la presencia de factores de confusión al comparar las estimaciones del nuevo modelo con las del modelo anterior. La confusión se consideró presente cuando las estimaciones cambiaron al menos en $25 \%$. Los factores de confusión fueron incluidos en el modelo independientemente de su importancia para obtener estimaciones menos sesgadas. La bondad de ajuste de los modelos se verificó mediante la prueba de estadística Hosmer-Lemeshow. La sensibilidad y especificidad del modelo fueron 82.9 y $83.3 \%$, respectivamente. Asimismo, los valores de $\mathrm{pH}_{24}$ se analizaron como una variable de respuesta binaria con valores de $\mathrm{pH}_{24}<6.1{\text { y } \mathrm{pH}_{24}}$ $>6.1$ y se hizo un análisis de regresión logística multivariante.

\section{Resultados}

En el análisis de regresión logística univariable se identificaron diferencias significativas entre los casos y los controles para nueve variables (Cuadros 1 y 2). El modelo de regresión logística mostró que el número de compartimientos del camión, la presencia de un ayudante durante el viaje, el sexo de los cerdos, la velocidad y el tiempo de transporte fueron variables asociadas con la presencia de cerdos fatigados $(\mathrm{p}<0.05)$ (Cuadro 3).

Las muestras de carne se distribuyeron en los siguientes grupos de calidad: canales con $\mathrm{pH}_{45} \leq 5.8$ fueron consideradas como PSE, canales con $\mathrm{pH}_{24}$ entre 5.6 y 6.0 como de calidad normal y $\mathrm{pH}_{24}>6.1$ se consideró como DFD (Boler et al., 2010). La mayoría de los lomos $(75 \%)$ evaluados en el estudio se clasificaron como DFD. Así mismo, se encontró un porcentaje menor de PSE (5.3\%). La carne con $\mathrm{pH}_{45}$ normal se encontró en una proporción de $19.8 \%$. Los cerdos fatigados presentaron mayor frecuencia de carne oscura que los cerdos típicos $(\mathrm{OR}=4.7 ; \mathrm{p}<0.01)$.

\section{Discusión}

Aunque generalmente se acepta que los viajes más largos pueden afectar negativamente el bienestar animal y las pérdidas por transporte de los cerdos de ceba (Haley et al., 2008; Sutherland et al., 2009a), algunos estudios (Pérez et al., 2002; Pilcher et al., 2011) reportan una mayor prevalencia de cerdos fatigados en viajes inferiores a ocho horas, que es el valor crítico superior reportado en la legislación europea y colombiana en los viajes categorizados como largos (Reglamento del Consejo Europeo (CE), 2004; MPS, 2007). El presente estudio encontró que la presencia de cerdos fatigados se 
Cuadro 1. Análisis univariable de variables asociadas a la presencia de cerdos fatigados en un estudio de casos y controles, en una planta de sacrificio comercial en Colombia (Parte 1)

\begin{tabular}{|c|c|c|c|c|c|}
\hline Variables & $\begin{array}{l}\text { Caso } \\
\mathrm{n}(\%)\end{array}$ & $\begin{array}{c}\text { Control } \\
\mathrm{n}(\%)\end{array}$ & $\begin{array}{c}P \\
\text { value }\end{array}$ & OR & IC 95\% \\
\hline \multicolumn{6}{|l|}{$\begin{array}{l}\text { Características del } \\
\text { conductor }\end{array}$} \\
\hline \multicolumn{6}{|l|}{ Formación en bienestar animal } \\
\hline Sí & $85(90.4)$ & $9(9.6)$ & 0.625 & 0.74 & $0.23-2.42$ \\
\hline No & $75(50.0)$ & $75(50.0)$ & & & \\
\hline \multicolumn{6}{|l|}{ Edad (años) } \\
\hline $30-40$ & $32(34.1)$ & $73(48.7)$ & 0.644 & 0.85 & $0.43-1.68$ \\
\hline $41-50$ & $57(60.6)$ & $69(46.0)$ & & & \\
\hline $51-60$ & $5(5.3)$ & $8(5.3)$ & & & \\
\hline \multicolumn{6}{|l|}{ Escolaridad } \\
\hline Primaria & $9(9.6)$ & $5(3.3)$ & 0.06 & 0.35 & $0.12-1.04$ \\
\hline Bachillerato & $84(89.4)$ & $129(86.0)$ & & & \\
\hline Técnico & $1(1.0)$ & $16(10.7)$ & & & \\
\hline \multicolumn{6}{|l|}{ Condiciones de viaje } \\
\hline \multicolumn{6}{|l|}{$\begin{array}{l}\text { Tamaño del grupo en el } \\
\text { camión (n) }\end{array}$} \\
\hline $3-20$ & $3(3.2)$ & $86(57.3)$ & & & \\
\hline $21-40$ & $25(26.6)$ & $34(22.7)$ & & & \\
\hline $50-80$ & $45(47.9)$ & $26(17.3)$ & & & \\
\hline $100-120$ & $21(22.3)$ & $4(2.7)$ & & & \\
\hline $\begin{array}{l}\text { Densidad de carga }(0.52-0.8 \\
\mathrm{m}^{2} / \text { cerdo) }\end{array}$ & $94(100)$ & $143(95.3)$ & 0.08 & 0.00 & $0.00-2.01$ \\
\hline \multicolumn{6}{|l|}{ Distancia $(\mathrm{km})$} \\
\hline $10-50$ & $13(13.8)$ & $77(51.3)$ & 0.05 & 2.54 & $1.0-6.49$ \\
\hline $51-100$ & $81(86.2)$ & $73(48.7)$ & & & \\
\hline \multicolumn{6}{|l|}{ Tiempo de transporte $(\mathrm{h})$} \\
\hline $0.3-1$ & $3(3.2)$ & $19(12.7)$ & 0.01 & 0.55 & $0.34-0.90$ \\
\hline $1.1-2$ & $38(40.4)$ & $67(44.7)$ & & & \\
\hline $2.1-3$ & $52(55.3)$ & $37(24.6)$ & & & \\
\hline $3.1-4$ & $1(1.0)$ & $27(18.0)$ & & & \\
\hline \multicolumn{6}{|l|}{ Velocidad $(\mathrm{km} / \mathrm{h})$} \\
\hline $30-60$ & $80(85.1)$ & $138(92.0)$ & 0.65 & 0.75 & $0.22-2.56$ \\
\hline $61-80$ & $14(14.9)$ & $12(8.0)$ & & & \\
\hline Ayudante (Sí) & $26(27.7)$ & $43(28.7)$ & $<0.01$ & 0.26 & $0.11-0.59$ \\
\hline Preclasificación (Sí) & $10(10.6)$ & $30(20.0)$ & 0.88 & 0.93 & $0.35-2.45$ \\
\hline Mezcla de cerdos (No) & $89(94.7)$ & $104(69.3)$ & 0.88 & 0.93 & $0.35-2.45$ \\
\hline Paradas durante el viaje (Sí) & $91(96.8)$ & $103(68.7)$ & 0.56 & 1.52 & $0.36-6.35$ \\
\hline Carretera mixta (Sí) & $86(91.5)$ & $146(97.3)$ & 0.02 & 0.37 & $0.16-0.84$ \\
\hline Suministro de agua (Sí) & $78(83.0)$ & $48(32.0)$ & 0.54 & 0.72 & $0.25-2.05$ \\
\hline
\end{tabular}

${ }^{1}$ OR: Razón de disparidad; ${ }^{2} \mathrm{Cl}=$ intervalo de confianza 
Cuadro 2. Análisis univariable de variables asociadas a la presencia de cerdos fatigados en un estudio de casos y controles, en una planta de sacrificio comercial en Colombia (Parte 2)

\begin{tabular}{|c|c|c|c|c|c|}
\hline Variables & $\begin{array}{l}\text { Caso } \\
\mathrm{n}(\%) \\
\end{array}$ & $\begin{array}{c}\text { Control } \\
\mathrm{n}(\%)\end{array}$ & $\begin{array}{c}P \\
\text { value }\end{array}$ & $\mathrm{OR}^{1}$ & $\mathrm{IC}^{2} 95 \%$ \\
\hline \multicolumn{6}{|l|}{ Características del camión } \\
\hline \multicolumn{6}{|l|}{ Número de pisos } \\
\hline Uno & $6(6.4)$ & $102(68.0)$ & $<0.01$ & 2.14 & $1.63-29.1$ \\
\hline Dos & $88(93.6)$ & $48(32.0)$ & & & \\
\hline \multicolumn{6}{|l|}{ Piso } \\
\hline Aluminio & 75 (79.7) & $50(33.3)$ & 0.88 & 0.95 & $0.54-1.68$ \\
\hline Madera & $19(20.3)$ & $100(66.7)$ & & & \\
\hline Techo de lona (Sí) & $35(37.2)$ & $92(61.3)$ & 0.03 & 2.58 & $1.09-6.08$ \\
\hline Compartimentos (Sí) & $83(88.3)$ & $71(47.3)$ & 0.94 & 1.03 & $0.37-2.87$ \\
\hline \multicolumn{6}{|l|}{ Otras variables } \\
\hline Género & $49(52.1)$ & $95(63.3)$ & 0.03 & 2.02 & $1.02-3.98$ \\
\hline Cerdos muertos a la llegada (n) & 3 & 5 & 0.81 & 0.89 & $0.35-2.25$ \\
\hline
\end{tabular}

${ }^{1} \mathrm{OR}$ : Razón de disparidad; ${ }^{2} \mathrm{Cl}=$ intervalo de confianza

Cuadro 3. Factores asociados a la presencia de cerdos fatigados según los resultados de la regresión logística multivariable (94 casos y 94 controles)

\begin{tabular}{lcccccc}
\hline Variable & Categoría & OR & Coef. & IC 95\% & $\begin{array}{c}p \text { value } \\
\text { Test Wald }\end{array}$ value \\
\hline Tamaño del grupo en & $3-20$ & Ref. & & & & NS \\
el camión (n) & $21-40$ & 5.5 & 1.7 & $0.3-3.6$ & & NS \\
& $50-80$ & 9.0 & 2.2 & $0.2-3.5$ & $<0.01$ & 0.01 \\
& $100-120$ & 34.5 & 3.5 & $1.6-6.3$ & & 0.02 \\
$\begin{array}{l}\text { Tiempo de transporte } \\
\text { (h) }\end{array}$ & Numérico & 0.48 & -1.2 & $0.3-0.9$ & 0.02 & \\
Número de pisos & Uno & Ref. & & $1.3-3.3$ & $<0.01$ & $<0.01$ \\
& Dos & 6.9 & 1.5 & & & \\
Velocidad (km/h) & Numérico & 1.0 & 0.07 & $0.0-0.1$ & 0.02 & $<0.01$ \\
Asistente & Sín & 0.2 & & $-0.1-0.6$ & $<0.01$ & $<0.01$ \\
& No & Ref. & -1.8 & & & \\
Género & $\begin{array}{l}\text { Cerdos } \\
\text { castrados }\end{array}$ & Ref. & & & & \\
& $\begin{array}{c}\text { Cérdas } \\
\text { jóvenes }\end{array}$ & 2.3 & 0.8 & $0.1-1.1$ & 0.04 & 0.03 \\
\hline
\end{tabular}

$\mathrm{OR}=$ razón de disparidad; $\mathrm{Cl}$ = intervalo de con? anza; Ref: categoría considerada como referencia; NS: no significativo 
incrementó en viajes de corta duración y disminuyó en transportes de mayor duración debido, tal vez, a la falta de recuperación de los cerdos al estrés experimentado durante los procedimientos de embarque en la granja y desembarque en la planta como ha sido descrito por Ritter et al. (2006) en EEUU y por Romero-Peñuela et al. (2015) en Colombia. Por otro lado, en este estudio, la mayor proporción de camiones presentó densidades de carga consistentes con la legislación nacional (ICA, 2007), por lo que esta variable no se asoció con la presencia de cerdos fatigados. Se necesitan estudios adicionales para explicar la relación entre las condiciones del viaje y las pérdidas por transporte en los cerdos de ceba.

Los cerdos son transportados en Colombia en camiones con diseño y tamaños variados, dotados de sistemas de ventilación pasiva; así mismo, el nivel de capacitación de los conductores en manejo animal es bajo (Romero et al., 2016), por lo que el tipo de camión puede afectar el comportamiento de los cerdos, además del transporte, el desembarque y el manejo en la planta de sacrificio (Torrey et al., 2013). Debido a razones económicas, el uso de camiones de dos y tres pisos para el transporte de cerdos está aumentando rápidamente en Colombia (Romero et al., 2016). Sin embargo, solo un bajo porcentaje de los modelos están equipados con rampas hidráulicas que faciliten el embarque y desembarque de los animales. El número de pisos del camión tuvo efecto sobre la presencia de cerdos fatigados en este estudio, lo que concuerda con estudios previos (Romero et al. 2016). El embarque y desembarque de cerdos a través de rampas impone un fuerte esfuerzo físico para los cerdos (Goumon et al., 2013), que se aumenta por la intervención aversiva de los manejadores, lo cual resulta en un mayor esfuerzo físico para los cerdos y el personal (Torrey et al., 2013).

En el presente estudio, la velocidad del camión fue un factor que aumentó la probabilidad de presentar cerdos fatigados durante el transporte. Las variaciones en la velocidad durante el transporte causan vibración y pérdida de equilibrio (Gebresenbet et al., 2011) y los cerdos son sensibles a estas dos condiciones (Li et al., 2008). Cuando se exponen a la vibración, los órganos corporales funcionan como un grupo heterogéneo de sistemas mecánicos que causa el desplazamiento de los órganos internos, lo cual produce estrés fisiológico y conductual en los animales ( $\mathrm{Lu}$ et al., 2010; Gebresenbet et al., 2011).

Las cerdas jóvenes evaluadas presentaron mayor riesgo de fatiga posiblemente porque son más susceptibles al estrés que los cerdos castrados (Pérez et al., 2002). No obstante, otros autores indican que los machos tienen encuentros antagónicos para establecer nuevas jerarquías (Lambooij, 2007; Sutherland et al., 2008). Las diferencias entre las tasas metabólicas o las reservas de energía entre machos y hembras pueden contribuir a los efectos observados en la presencia de cerdos fatigados. Estudios demuestran que mantener los cerdos separados por sexo en camiones mixtos podría reducir el porcentaje de animales muertos y fatigados (Sutherland et al., 2009b).

En este estudio se evidenció que la presencia de un asistente o ayudante durante el viaje fue un factor que disminuyó la presencia de cerdos fatigados. No obstante, la baja proporción de camiones con la presencia de un asistente en el estudio refuerza la necesidad de implementar esta medida, pero es indispensable contar con personal entrenado.

Dependiendo del intervalo de tiempo entre la inducción de la fatiga de los cerdos y el sacrificio, el animal podrá o no agotar sus reservas de glucógeno muscular antes de la muerte (Carr et al., 2005). En este estudio, los resultados mostraron que los cerdos fatigados presentaron una mayor prevalencia de carne con $\mathrm{pH}$ alto que los cerdos típicos. Estos resultados, son similares a estudios previos (Hambrecht et al., 2005; Weschenfelder et al., 2012; Correa et al., 2013) y confirman que los cerdos fatigados experimentan más 
estrés antes del sacrificio, comparados con los cerdos de ceba típicos, y que los efectos del estrés en la calidad de la carne son dependientes del músculo (Correa et al., 2013). El esfuerzo físico y el estrés psicológico aumentan la secreción de hormonas que exacerban los efectos de la actividad muscular sobre el agotamiento del glucógeno muscular (Terlouw y Porcher, 2005). Por otro lado, un pequeño porcentaje de cerdos exhibió carne PSE, lo cual puede estar relacionado con un agotamiento de las reservas de glucógeno en un corto plazo, justo antes del sacrificio (Carr et al. 2005).

\section{Conclusiones}

- Se identificó que el tiempo de transporte, la velocidad, el número de pisos del camión, la ausencia de un asistente durante el viaje y el sexo de los cerdos son factores determinantes para la presencia de cerdos fatigados.

- Los cerdos fatigados tuvieron un mayor riesgo de presentar carne con $\mathrm{pH}$ alto al compararlos con los cerdos típicos.

- La presencia de cerdos fatigados durante el transporte es un indicador útil para evaluar el bienestar animal, ya que esta condición compromete la homeostasis del animal, su capacidad para adaptarse a las condiciones estresantes del pre-sacrificio y afecta de manera negativa la calidad de la carne.

\section{Literatura Citada}

1. Aradom S, Gebresenbet G, Bullita FS, Bobobee EY, Adam M. 2012. Effect of transport times on welfare of pigs. J Agric Sci Technol 2: 544-562.

2. Berry NL, Johnson AK, Hill J, Lonergan S, Karriker LA, Stalder KJ. 2012. Loading gantry versus traditional chute for the finisher pig: effect on welfare at the time of loading and performance measures and transport losses at the harvest facility. J Anim Sci 90: 4028-4036. doi: 10.2527/jas.2011-4973

3. Boler DD, Dilger AC, Bidner BS, Carr SN, Eggert JM, Day JW, Ellis M, et al. 2010. Ultimate $\mathrm{pH}$ explains variation in pork quality traits. J Muscle Foods 21: 119-130. doi: 10.1111/j.1745-4573.2009.00171.x

4. Carr SN, Gooding JP, Rincker PJ, Hamilton DN, Ellis M, Killefer J, Mckeith FK. 2005. A survey of pork quality of downer pigs. J Muscle Foods 16: 298-305. doi: $10.1111 / \mathrm{j} .1745-$ 4573.2005.00022. $\mathrm{x}$

5. Correa JA, Gonyou $H W$, Torrey $S$, Widowski T, Bergeron R, Crowe TG, Laforest JP, Faucitano L. 2013. Welfare and carcass and meat quality of pigs being transported for two hours using two vehicle types during two seasons of the year. Can J Anim Sci 93: 43-55. doi: 10.4141/cjas2012-088

6. European Council Regulation (EC) 2004. Council Regulation (EC) No 1/ 2005 of 22 December 2004 on the protection of animals during transport and related operations and amending Directives 64/432/EEC and 93/119/EC and Regulation (EC) No 1255/97. [Internet]. Available in: https://eurlex.europa.eu/eli/reg/2005/1/oj

7. [FAOSTAT] Online database of the Food and Agriculture Organization of the United Nations. sf. [Internet] Available in: http://www.fao.org/faostat/ en/\#data/QL

8. Gajana CS, Nkukwana TT, Marume U, Muchenje V. 2013. Effects of transportation time, distance, stocking density, temperature and lairage time on incidences of pale soft exudative (PSE) and the physico-chemical characteristics of pork. Meat Sci 95: 520-525. doi: 10.1016/j.meatsci.2013.05.028

9. Gebresenbet G, Aradom S, Bulitta FS, Hjerpe E. 2011. Vibration levels and frequencies on vehicle and animals during transport. Biosyst Eng 110: 10-19. doi: 10.1016/j.biosystemseng.2011.05.-007 
10. Goumon S, Brown JA, Faucitano L, Bergeron R, Widowski TM, Crowe T, Connor ML, et al. 2013. Effects of transport duration on maintenance behavior, heart rate and gastrointestinal tract temperature of market-weight pigs in 2 seasons. J Anim Sci 91: 4925-4935. doi: $10.2527 /$ jas.2012-6081

11. Haley C, Dewey CE, Widowski T, Poljak Z, Friendship R. 2008. Factors associated with in-transit losses of market hogs in Ontario in 2001. Can J Vet Res 72: 377-384

12. Hambrecht E, Eissen JJ, Newman DJ, Smits CHM, Den Hartog LA, Verstegen MWA. 2005. Negative effects of stress immediately before slaughter on pork quality are aggravated by suboptimal transport and lairage conditions. J Animal Sci 83: 440-448. doi 10.2527/2005.832440x

13. [ICA] Instituto Colombiano Agropecuario. 2007. Resolución 002640 de 28 de septiembre de 2007. [Internet], Disponible en: https://www.ica.gov.co/ getattachment/6bfd1517-10f1-415db8cd-3ccb06d51a8f/2640.aspx

14. Johnson AK, Gesing LM, Ellis M, McGlone JJ, Berg E, Lonergan SM, Fitzgerald R, et al. 2013. 2011 and 2012 Early Careers Achievement Awards: farm and pig factors affecting welfare during the marketing process. J Anim Sci 91: 24812491. doi: $10.2527 /$ jas.2012-6114

15. Kephart KB, Harper MT, Raines $C R$. 2010. Observations of market pigs following transport to a packing plant. J Anim Sci 88: 2199-2203. doi: 10.2527/ jas.2009-2440.

16. Lambooij B. 2007. Transport of pigs. In: Grandin T (eds). Livestock handling and transport. $3^{\text {rd }}$ Rev ed. Colorado, USA: CABI Publishing. p 228-244.

17. Li LA, Xia D, Bao ED, Wei S, Xiao JS, Bao JW, Chen WH, et al. 2008. Erhualian and Pietrain pigs exhibit distinct behavioral, endocrine and biochemical responses during transport. Livest Sci 113: 169-177. doi: 10.1016/j.livsci.2007.03.008
18. Lu F, Ishikawa Y, Kitazawa H, Satake T. 2010. Effect of vehicle speed on shock and vibration levels in truck transport. Packag Technol Sci 23: 101109. doi: $10.1002 /$ pts. 882

19. Magnani D, Cafazzo S, Cal P, Razzuoli E, Amadori M, Bernardini D, Gerardi G, et al. 2014. Effect of long transport and environmental conditions on behaviour and blood parameters of postweaned piglets with different reactivity to backtest. Livest Sci 162: 201208. doi: 10.1016/j.livsci.2014.01.011

20. [MPS] Ministerio de la Protección Social. 2007. Decreto Número 1500 de 2007. [Internet], Disponible en: http:// www.minambiente.gov.co/images/normativa/decretos $/ 2007 /$ dec 1500 2007.pdf

21. Pérez MP, Palacio J, Santolaria MP, Aceña MDC, Chacón G, Verde MT, Calvo JH, et al. 2002. Influence of lairage time on some welfare and meat quality parameters in pigs. Vet Res 33: 239-250. doi: 10.1051/vetres:2002012

22. Pilcher CM, Ellis M, Rojo-Gómez A, Curtis SE, Wolter BF, Peterson CM, et al. 2011. Effects of floor space during transport and journey time on indicators of stress and transport losses of marketweight pigs. J Anim Sci 89: 3809-3818. doi: 10.2527/jas.2010-3143

23. Ritter MJ, Ellis M, Anderson DB, Curtis SE, Keffaber KK, Killefer J, McKeith FK, et al. 2009a. Effects of multiple concurrent stressors on rectal temperature, blood acid-base status, and longissimus muscle glycolytic potential in market-weight pigs. J Anim Sci 87: 351362. doi: $10.2527 /$ jas.2008-0874

24. Ritter MJ, Ellis M, Berry NL, Curtis SE, Anil L, Berg E, Benjamin M, et al. 2009b. Review: transport losses in market weight pigs: i. a review of definitions, incidence, and economic impact. Prof Anim Scientist 25: 404-414. doi: 10.15232/S1080-7446(15)30735-X

25. Ritter MJ, Ellis M, Brinkmann J, DeDecker JM, Keffaber KK, Kocher $M E$, Peterson BA, et al. 2006. Effect of floor space during transport of market- 
weight pigs on the incidence of transport losses at the packing plant and the relationships between transport conditions and losses. J Anim Sci 84: 2856-2864. doi: 10.2527/jas.2005-577

26. Romero MH, Sánchez JA, Hoyos R. 2016. Factores asociados con la frecuencia de cerdos no ambulatorios durante el transporte. Arch Med Vet 48: 191-198. doi: 10.4067/S0301-732X2016000200009

27. Romero-Peñuela MH, Sánchez-Valencia JA, Hoyos-Martínez RH. 2015. Factors associated with the frequency of died pigs during transport to a slaughterhouse. Rev CES Med Zootec 10: 132-140.

28. Sutherland MA, Erlandson K, Connor JF, Salak-Johnson JL, Matzat P, Smith JF, McGlone JJ. 2008. Health of non-ambulatory, non-injured pigs at processing. Livest Sci: 116: 237-245. doi: 10.1016/j.livsci.2007.10.009

29. Sutherland MA, Krebs N, Smith JS, Dailey JW, Carroll JA, McGlone JJ. 2009a. The effect of three space allowances on the physiology and behavior of weaned pigs during transpor- tation. Livest Sci 126: 183-188. doi: 10.1016/j.livsci.2009.06.021

30. Sutherland MA, McDonald A, McGlone JJ. 2009b. Effects of variations in the environment, length of journey and type of trailer on the mortality and morbidity of pigs being transported to slaughter. Vet Rec 165: 13-18.

31. Terlouw EM, Porcher J, Fernandez

$X$. 2005. Repeated handling of pigs during rearing. II. Effect of reactivity to humans on aggression during mixing and on meat quality. J Anim Sci 83: 16641672. doi: $10.2527 / 2005.8371664 \mathrm{x}$

32. Torrey S, Bergeron R, Widowski T, Lewis N, Crowe T, Correa JA, et al. 2013. Transportation of market-weight pigs: effect of season, truck type, and location within truck on behavior with a two-hour transport. J Anim Sci 91: 28632871. doi: $10.2527 /$ jas.2012-6005

33. Weschenfelder AV, Torrey S, Devillers $N$, Crowe T, Bassols A, Saco Y, Pineiro $M$, et al. 2012. Effects of trailer design on animal welfare parameters and carcass and meat quality of three Pietrain crosses being transported over a long distance. J Anim Sci 90: 32203231. doi: $10.2527 /$ jas.2012-4676 